\title{
Chiral dynamics of few-nucleon systems
}

\author{
E. Epelbaum \\ Forschungszentrum Jülich, Institut für Kernphysik, D-52425 Jülich, Germany \\ Universität Bonn, Helmholtz-Institut für Strahlen- und Kernphysik, D-53115 Bonn, Germany
}

\begin{abstract}
I discuss some recent developments in chiral effective field theory for few-nucleon systems.
\end{abstract}

Key words: Chiral effective field theory, nuclear forces, few-nucleon systems

PACS: 13.75.Cs, 21.30.-x, 21.45.+v

\section{Introduction}

Almost two decades ago Weinberg formulated his ideas to extend chiral perturbation theory to systems with several nucleons [1]. This seminal work initiated extensive research towards better understanding of the structure of nuclear forces and the dynamics of few- and many-nucleon systems based on the powerful framework of effective field theory (EFT). In this talk I outline the current status of chiral EFT for nuclear forces and applications in the few-nucleon sector. I discuss the structure of the subleading contributions to the three-nucleon force $(3 \mathrm{NF})$ and the leading four-nucleon force (4NF) which are currently being investigated. The role of $\Delta$-excitation will also be addressed. The last part of this talk concerns lattice simulations of light nuclei.

\section{Chiral EFT for few nucleons: present status}

Chiral EFT is an appropriate framework to analyze the properties of few-nucleon systems at low energy 1 It is based on the most general effective Lagrangian for Goldstone bosons (pions) and matter fields (nucleons and perhaps deltas) consistent with the chiral symmetry of QCD. For energies below the pion-production threshold, a nonrelativistic

Email address: e.epelbaum@fz-juelich.de (E. Epelbaum).

1 At very low energies, it is advantageous to use pionless EFT, see [2] for a recent review article. 
treatment of the nucleons is justified. Further, it is advantageous to eliminate the pionic degrees of freedom which gives rise to the (nonlocal) nuclear Hamiltonian

$$
H_{\text {nucl }}=H_{0}+V_{2 N}+V_{3 N}+V_{4 N}+\ldots
$$

The resulting quantum-mechanical $A$-body problem can be dealt with using rigorous few-body techniques or many-body methods.

The derivation of the nuclear potentials from the effective Lagrangian can be carried out straightforwardly utilizing the so-called chiral expansion. The importance of a particular contribution to the nuclear Hamiltonian is determined by the corresponding power of the expansion parameter $Q / \Lambda$ where $Q$ and $\Lambda$ refer to the generic low-momentum scale associated with external nucleon momenta or $M_{\pi}$ and the pertinent hard scale, respectively [1]. For the two-nucleon force $(2 \mathrm{NF})$, this expansion has the form

$$
V_{2 N}=V_{2 N}^{(0)}+V_{2 N}^{(2)}+V_{2 N}^{(3)}+V_{2 N}^{(4)}+\ldots,
$$

where the subscripts refer to the chiral order. The leading contribution $V_{2 N}^{(0)}$ is due to $1 \pi$ exchange (OPE) and two NN contact interactions without derivatives. The corrections result from contact interactions with increasing number of derivatives and/or insertions of $M_{\pi}^{2}$ and exchange of pions. In particular, $2 \pi$-exchange starts to contribute at nextto-leading order (NLO) and provides an important ingredient of the nuclear force [3]. At present, the $2 \mathrm{NF}$ has been worked out and applied in the NN system at next-tonext-to-next-to-leading order $\left(\mathrm{N}^{3} \mathrm{LO}\right)[4,5]$, see [6] for the pioneering work along this line. Its long-range part involves contributions from $1 \pi$ - $2 \pi$ - and $3 \pi$-exchange and is parameter-free since the corresponding low-energy constants (LECs) are known from $\pi N$ scattering. Numerically, the $3 \pi$-exchange potential turns out to be negligibly small [7]. The short-range part consists of 24 independent contact interactions 2 whose strengths were determined from fits to NN low-energy data, see Ref. [8] for the discussion on resonance saturation of the corresponding LECs. Both $\mathrm{N}^{3} \mathrm{LO}$ calculations of Ref. [4] and [5] yield compatible results for the NN system (within the theoretical uncertainty) and demonstrate an accurate description of the low-energy scattering data and the deuteron properties.

Systems with three- and more nucleons provide an excellent testing ground for chiral nuclear forces and were extensively studied in this framework over the past few years. Chiral power counting explains naturally the observed hierarchy of nuclear forces $V_{2 N} \gg$ $V_{3 N} \gg V_{4 N} \ldots$ with

$$
V_{3 N}=V_{3 N}^{(3)}+V_{3 N}^{(4)}+\ldots, \quad V_{4 N}=V_{3 N}^{(4)}+V_{3 N}^{(5)}+\ldots, \ldots .
$$

The first nonvanishing contribution to the $3 \mathrm{NF}$ appears at $\mathrm{N}^{2} \mathrm{LO}$ relative to the leading $2 \mathrm{NF}$ and results from $2 \pi$-exchange, $1 \pi$-exchange with $2 \mathrm{~N}$ contact interaction and the purely short-range $3 \mathrm{~N}$ contact term. While the $2 \pi$-exchange contribution is parameterfree, the two other topologies depend on the unknown LECs $D$ and $E$ which were fitted to the ${ }^{3} \mathrm{H}$ binding energy and $n d$ doublet scattering length [9] and to the combinations of the ${ }^{3} \mathrm{H} /{ }^{4} \mathrm{He}[10]$ and ${ }^{3} \mathrm{H} /{ }^{10} \mathrm{~B}[11]$ binding energies. The resulting nuclear Hamiltonian was extensively tested in the $3 \mathrm{~N}$ continuum $[12,13,14,15]$ and applied to study the properties

2 This number does not include isospin-breaking terms and refers to Weinberg's counting rules based on the naive dimensional analysis. Alternative counting schemes are currently being explored. 

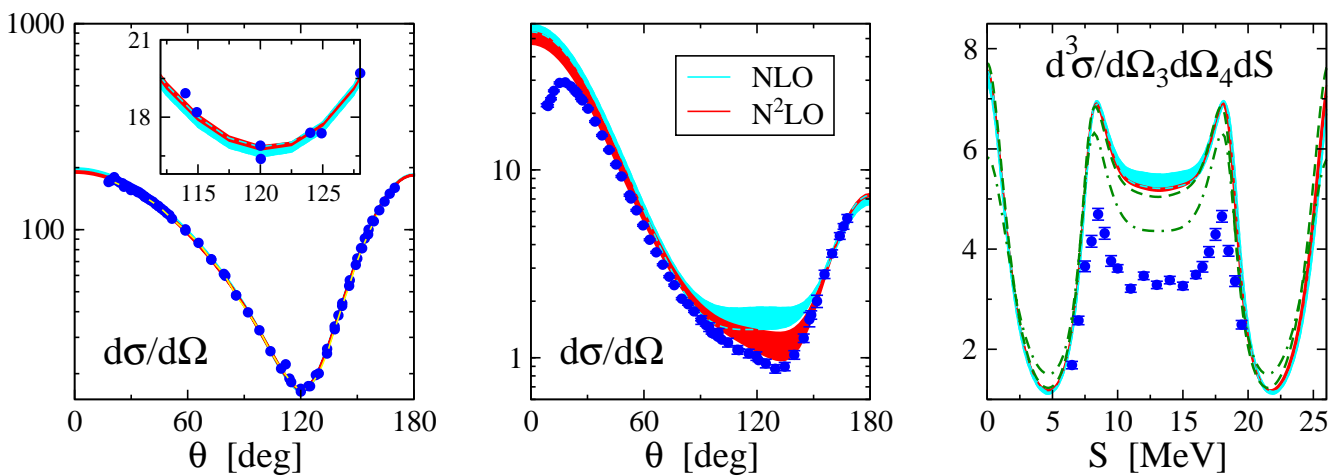

Fig. 1. Differential cross section (in mb/sr) for elastic $N d$ scattering at $10 \mathrm{MeV}$ (left panel) and $65 \mathrm{MeV}$ (middle panel) at NLO (light-shaded bands) and $\mathrm{N}^{2} \mathrm{LO}$ (dark-shaded bands). References to data can be found in [9]. Right panel shows $p d$ breakup cross section (in $\mathrm{mb} \mathrm{MeV}^{-1} \mathrm{sr}^{-2}$ ) along the kinematical locus $\mathrm{S}$ at $19 \mathrm{MeV}$ nucleon energy in the SCRE configuration with $\alpha=56^{\circ}$ [12]. Dashed and dashed-dotted lines are results based on the CD Bonn 2000 2NF [17] combined with the TM99 3NF [18] and the coupled channel calculation including the explicit $\Delta$ and the Coulomb interaction [19], respectively.

of light nuclei using the no-core shell model approach $[10,11,16]$. The calculated $3 \mathrm{~N}$ scattering observables are generally in a reasonable agreement with the data which improves when going from NLO to $\mathrm{N}^{2} \mathrm{LO}$, see e.g. the left and middle panels in Fig. 1. Notice however that the theoretical uncertainty at $\mathrm{N}^{2} \mathrm{LO}$ appears to be rather large already at moderate energies, see the middle panel in Fig. 1. We further emphasize that there are certain observables for which remarkably large discrepancies with the data are found even at very low energy. A well-known example is the so-called symmetric space-star (SST) $N d$ breakup configuration at $E_{N}=13 \mathrm{MeV}[20,9]$, in which the plane in the c.m. system spanned by the outgoing nucleons is perpendicular to the beam axis. Recently, $p d$ data for a similar symmetric constant relative-energy configuration have been measured at $E_{d}=19 \mathrm{MeV}$ [12]. This geometry is characterized by the angle $\alpha$ between the beam axis and the plane in the c.m. system spanned by the outgoing nucleons. Similar to the SST geometry, one observes large deviations between the theory and the data, see right panel in Fig. 13 which also hold for calculations based on modern phenomenological potentials. The included 3NFs have little effect on the cross section while the effect of the Coulomb interaction is significant but removes only a part of the discrepancy. For more details on these and related topics the reader is referred to Ref. [21], see also Ref. [22].

\section{3. $\mathrm{N}^{3} \mathrm{LO}$ contributions to three- and four-nucleon forces}

Although the results for few-nucleon observables already look quite promising at $\mathrm{N}^{2} \mathrm{LO}$, it is mandatory to extend these studies to $\mathrm{N}^{3} \mathrm{LO}$ as it has already been done for the $2 \mathrm{~N}$ system. This would allow one to test the convergence of the chiral expansion and might shed some light on the long-standing puzzles in the $3 \mathrm{~N}$ continuum. The extension to

$\overline{3}$ We emphasize that chiral EFT results are obtained without taking into account Coulomb interaction. 


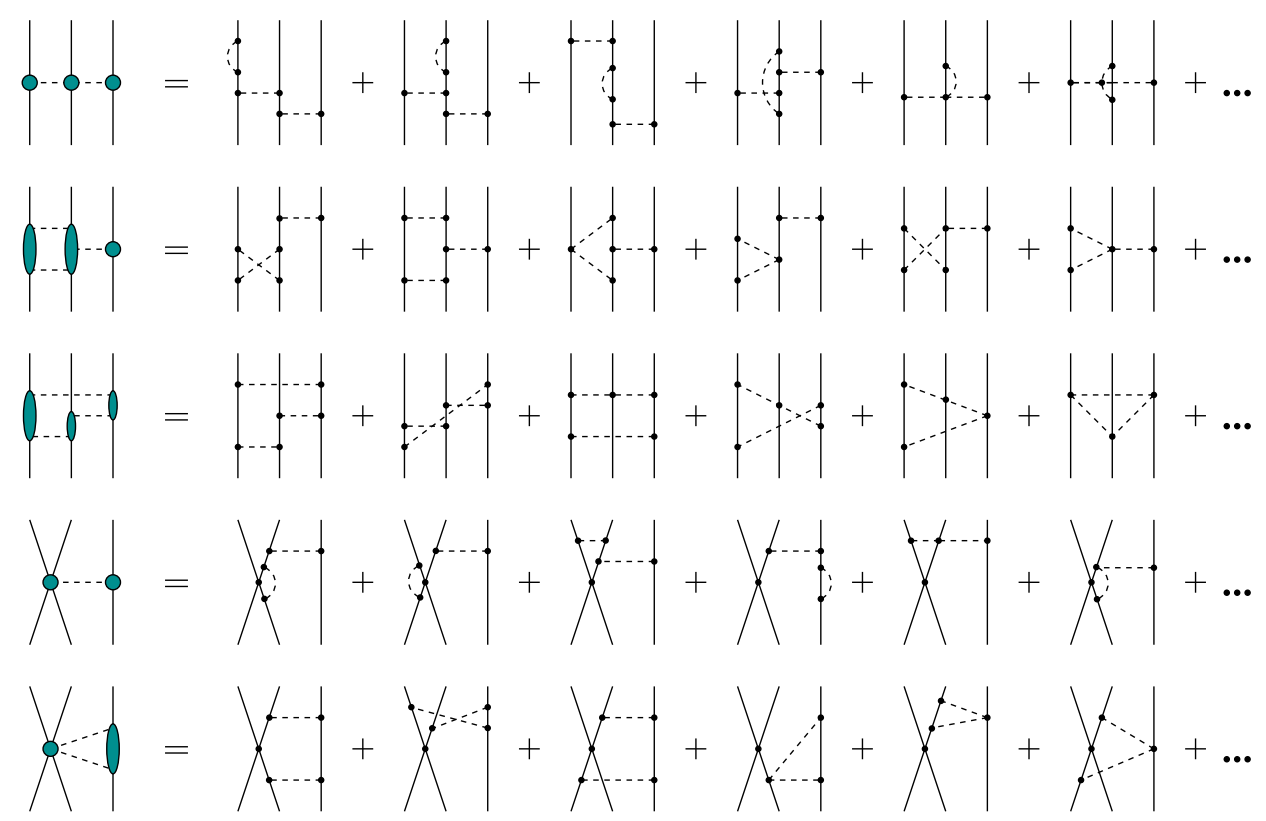

Fig. 2. Different $3 \mathrm{NF}$ contributions at $\mathrm{N}^{3} \mathrm{LO}$. Solid and dashed lines represent nucleons and pions, respectively. Solid dots denote the leading-order vertices.

$\mathrm{N}^{3} \mathrm{LO}$ requires the incorporation of the leading corrections to the $3 \mathrm{NF}$ which feed into five different topologies, see Fig. 2, and are currently being worked out. Interestingly, there are no contributions from higher-order vertices in the effective Lagrangian (except $1 / m$-corrections) so that the $3 \mathrm{NF}$ at $\mathrm{N}^{3} \mathrm{LO}$ is parameter-free. Notice further that the spin-space structure of the chiral $3 \mathrm{NF}$ at $\mathrm{N}^{3} \mathrm{LO}$ is much richer than the one of the currently available $3 \mathrm{NF}$ models. Partial results are already available for certain types of contributions. In particular, one-loop and relativistic $1 / m$-corrections to the $2 \pi$-exchange topology were considered in Refs. [23] and [24], respectively. The results of Ref. [23] based on the AV18 2NF [25] indicate that the one-loop $2 \pi$-exchange corrections have little effect on $3 \mathrm{~N}$ scattering observables.

In addition to the $3 \mathrm{NF}$ corrections, four-nucleon force $(4 \mathrm{NF})$ starts to contribute at $\mathrm{N}^{3} \mathrm{LO}$ in the chiral expansion. It has been worked out recently using the method of unitary transformation [26]. The leading $4 \mathrm{NF}$ results from diagrams depicted in Fig. 3 where only graphs are shown which yield nonvanishing contributions. It is governed by the exchange of pions and the lowest-order nucleon-nucleon contact interaction and includes effects due to the nonlinear pion-nucleon couplings and the pion self-interactions constrained by the chiral symmetry of QCD. The obtained 4NF is local and does not contain any unknown parameters. The expectation values of the individual $4 \mathrm{NF}$ contributions obtained in the pioneering study of Ref. [27] are typically of the order of few hundreds of $\mathrm{keV}$, which agrees with estimations based on dimensional arguments. One also observes strong cancellations between individual contributions. 

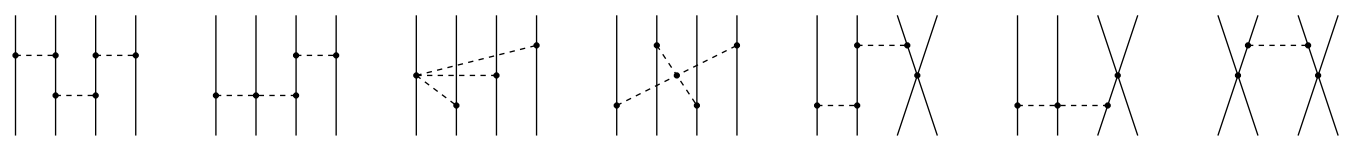

Fig. 3. $4 \mathrm{~N}$ diagrams generating nonvanishing $4 \mathrm{NF}$ contributions at $\mathrm{N}^{3} \mathrm{LO}$. Diagrams which result from the interchange of the nucleon lines and/or application of the time reversal operation are not shown. For remaining notation see Fig. 2.

\section{Role of $\Delta$-excitations}

The $\Delta$-isobar is known to play an important role in hadronic and nuclear physics due to its low excitation energy, $\Delta m \equiv m_{\Delta}-m=293 \mathrm{MeV}$, and strong coupling to the $\pi N$ system. It can, therefore, be argued that the explicit inclusion of the delta allows one to resum a certain class of important contributions and thus leads to an improved convergence as compared to the delta-less theory. Indeed, it is well known that e.g. the LECs $c_{3,4}$ accompanying the subleading $\pi \pi N N$ vertices are saturated by the $\Delta$-isobar which leads to their rather large numerical values [28]. As a consequence, the subleading $2 \pi$-exchange $2 \mathrm{NF}$ turns out to be much stronger than the leading one [29]. A similar tendency was also found for the $3 \pi$-exchange [7] and the charge-symmetry breaking $2 \pi$ exchange potentials [30]. In EFT with explicit deltas, the dominant portion of these large subleading contributions is shifted to the lower chiral order leading to a more natural convergence pattern.

The explicit inclusion of the delta in chiral EFT can be achieved via the so-called small scale expansion (SSE) [31] in which $\Delta m$ is treated as an additional small parameter. The leading contributions to the $2 \mathrm{NF}$ due to intermediate $\Delta$ excitations arise at NLO from diagrams shown in the first raw of Fig. 4 and were considered in [6,32]. In [33] we worked out the subleading contributions which are generated at $\mathrm{N}^{2} \mathrm{LO}$ by diagrams depicted in the second raw of Fig. 4. To obtain numerical results, one needs to determine the combination of the the subleading $\pi N \Delta$ LECs $b_{3}+b_{8}$ which enters at this order and the LECs $c_{i}$ whoes values differ from the ones obtained in the delta-less theory. This was achieved by fitting the threshold coefficients of $\pi N$ scattering calculated at second

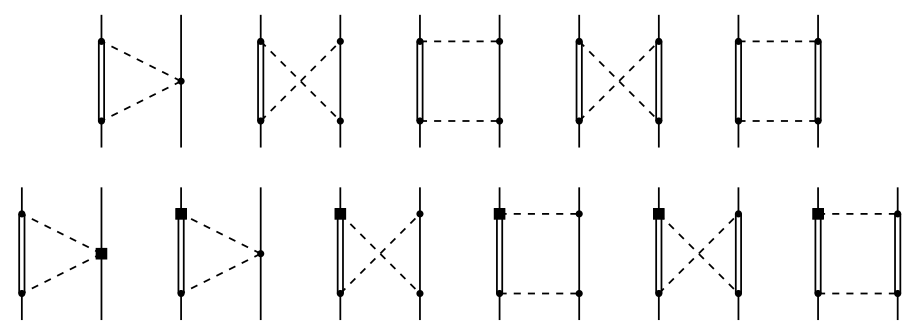

Fig. 4. NLO (first raw) and $\mathrm{N}^{2} \mathrm{LO}$ (second raw) contributions to the $2 \pi$-exchange $2 \mathrm{NF}$ with single and double $\Delta$ excitations. Double lines represent $\Delta$-isobars, filled squares denote subleading vertices. For remaining notation see Fig. 2. 
EFT with $\Delta$

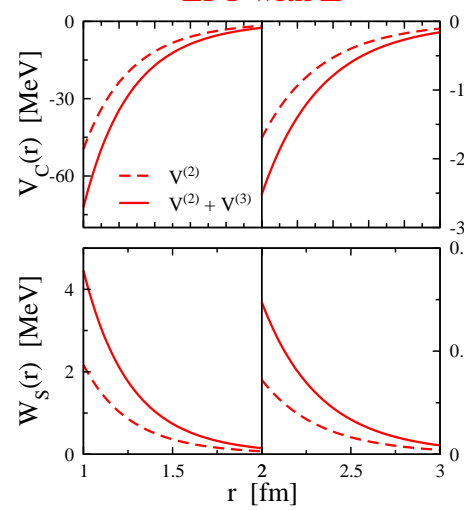

EFT without $\Delta$

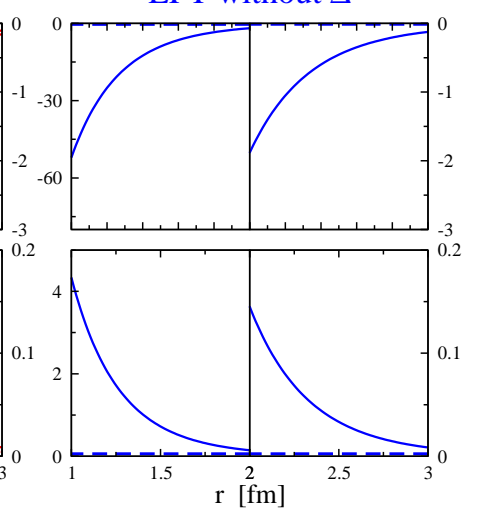

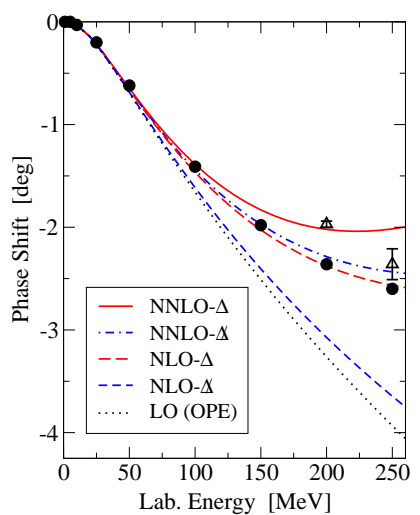

Fig. 5. Isoscalar central $\left(V_{C}(r)\right)$ and isovector spin-spin $\left(W_{S}(r)\right)$ potentials in $r$-space in EFT with (left panel) and without (middle panel) explicit $\Delta$ 's. Right panel: ${ }^{3} F_{3} \mathrm{NN}$ phase shift in EFT with and without explicit $\Delta$ 's calculated in first Born approximation. The filled circles (open triangles) depict the results from the Nijmegen multi-energy PWA [34] (Virginia Tech single-energy PWA [35]). In all cases, spectral-function regularization with the cutoff $\tilde{\Lambda}=700 \mathrm{MeV}$ has been used.

order in the SSE. As expected, we found that the values of the LECs $c_{3,4}$ are reduced in magnitude in the theory with explicit $\Delta$ 's. Using the $\mathrm{SU}(4)$ (or large $N_{c}$ ) value for the leading $\pi N \Delta$ LEC $h_{A}=3 g_{A} /(2 \sqrt{2})=1.34$ with $g_{A}$ being the nucleon axial-vector coupling, we found $c_{3}=-0.79 \mathrm{GeV}^{-1}$ and $c_{4}=1.33 \mathrm{GeV}^{-1}$ which has to be compared with $c_{3}=-3.87 \mathrm{GeV}^{-1}$ and $c_{4}=2.89 \mathrm{GeV}^{-1}$ in the delta-less EFT.

In Fig. 5 we compare the isoscalar central and isovector spin-spin $2 \pi$-exchange contributions at NLO and $\mathrm{N}^{2} \mathrm{LO}$ obtained in EFT with and without explicit $\Delta$ 's. Contrary to the delta-less theory where the whole contribution is generated at $\mathrm{N}^{2} \mathrm{LO}$ by subleading $2 \pi$-exchange, the dominant contribution in the delta-full theory results at NLO from the leading $2 \pi$-exchange graphs. This much natural convergence pattern is also observed for peripheral phase shifts, see Fig. 5 for an example.

\section{Few nucleons on a lattice}

The last topic I would like to address concerns nuclear lattice simulations using chiral EFT [36], see also [37] for a similar work based on pionless EFT. In this framework, the effective Lagrangian is formulated on a Euclidean lattice and the path integral is evaluated by Monte Carlo sampling. Pions and nucleons are treated as point-like particles on the lattice sites, and $\pi$ times the inverse lattice spacing sets the cutoff scale in momentum space. By using hadronic degrees of freedom and concentrating on low-energy physics, it is possible to probe larger volumes, lower temperatures, and far larger numbers of nucleons than in lattice QCD. In [36] simulations were carried out at lowest order in the chiral expansion. The sign problem is suppressed due to the SU(4) positivity of the lattice path integral for any even number of nucleons and the approximate SU(4) symmetry of the low-energy nuclear interactions. Having fixed three unknown parameters 

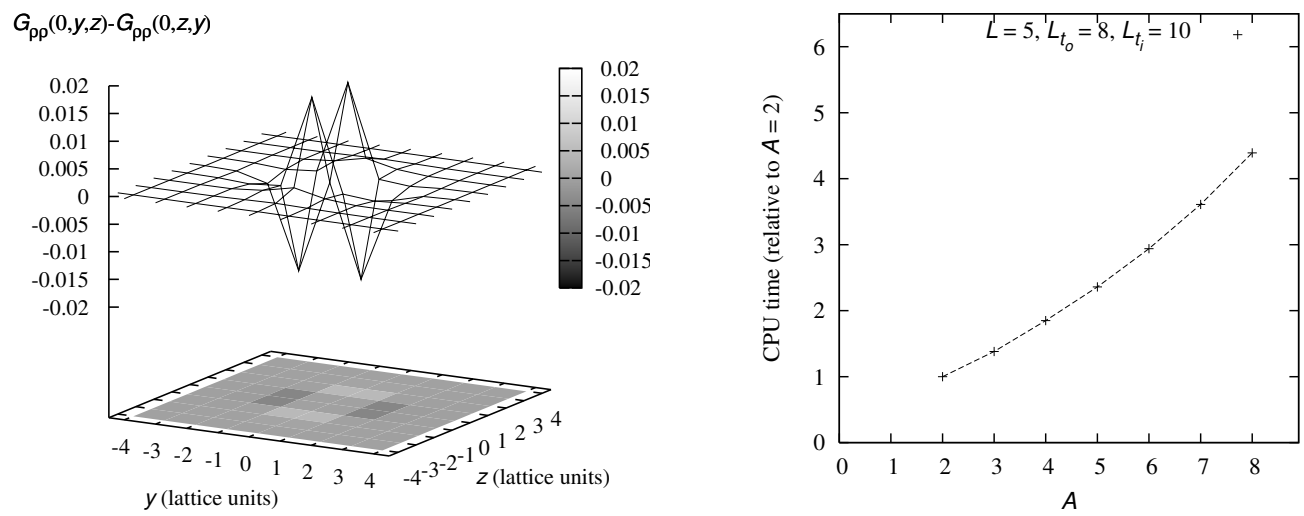

Fig. 6. Left panel: antisymmetric linear combination of the nucleon density correlations for the deuteron in the $y z$-plane. The deuteron spin points in the $+z$-direction. Right panel: CPU time versus the number of nucleons, $A$, measured relative to the $A=2$ deuteron system.

entering the $2 \mathrm{NF}$ using the ${ }^{2} \mathrm{H}$ binding energy, the $\mathrm{NN}{ }^{1} S_{0}$ scattering length and the average S-wave effective range 4 we computed various properties of light nuclei, see left panel in Fig. 6 for one example. The results for the deuteron properties and the obtained value for the triton binding energy $E_{3_{H}}=-8.9(2) \mathrm{MeV}$ are within $5 \%$ of the data while the $\alpha$-particle binding energy $E_{4}{ }_{H e}=-21.5(2) \mathrm{MeV}$ is about $25 \%$ smaller in magnitude than the experimental value of $-28.296 \mathrm{MeV}$. Encouraging results were also obtained for the computational scaling showing for $A \leq 8$ an approximately linear dependence on $A$, see right panel in Fig. 6.

\section{Summary and outlook}

In this talk I discussed the structure of the nuclear force in few lowest orders in the chiral expansion. In the $2 \mathrm{~N}$ system, accurate description of the low-energy data is achieved at $\mathrm{N}^{3} \mathrm{LO}$. The results for $3 \mathrm{~N}$ scattering and the properties of light nuclei are so far only available at $\mathrm{N}^{2} \mathrm{LO}$. While most of the calculated $3 \mathrm{~N}$ scattering observables are in a reasonable agreement with the data, the theoretical uncertainty at this order appears to be rather large. It is therefore mandatory to extend this studies to $\mathrm{N}^{3} \mathrm{LO}$. The corrections to the $3 \mathrm{NF}$ which arise at this order are currently being worked out. The leading $4 \mathrm{NF}$ which also contributes at $\mathrm{N}^{3} \mathrm{LO}$ has already been derived.

In the second part of my talk I discussed the $2 \mathrm{NF}$ due to intermediate $\Delta$-excitations at $\mathrm{N}^{2} \mathrm{LO}$ in the SSE. It is demonstrated that the explicit inclusion of the $\Delta$-isobar in chiral EFT allows to improve the convergence of the low-momentum expansion. In the future, this work should be extended to study the role of the $\Delta$-isobar in the $3 \mathrm{NF}$ and isospin-

4 The additional third parameter corresponds to higher-order contact interactions which were included to overcome a clustering instability, see [36] for more details. 
breaking interactions. It would also be interesting to work out $\mathrm{N}^{3} \mathrm{LO}$ contributions in the SSE.

Finally, in the last part of my talk, I discussed the leading-order results for the properties of light nuclei based on the lattice formulation of chiral EFT. A generalization to higher orders in the chiral expansion and to scattering observables, see [38] for the important step in this direction, is in progress.

Acknowledgements

I thank the organizers for the invitation and all my collaborators for sharing their insight into the discussed topics. This work was supported in parts by the Helmholtz Association under the contract number VH-NG-222.

\section{References}

[1] S. Weinberg, Phys. Lett. B251, 288 (1990).

[2] E. Braaten and H.-W. Hammer, Phys. Rept. 428, 259 (2006), cond-mat/0410417

[3] M. C. M. Rentmeester et al., Phys. Rev. Lett. 82, 4992 (1999), nucl-th/9901054

[4] D. R. Entem and R. Machleidt, Phys. Rev. C68, 041001 (2003), nucl-th/0304018

[5] E. Epelbaum, W. Glöckle, and U.-G. Meißner, Nucl. Phys. A747, 362 (2005), nucl-th/0405048

[6] C. Ordóñez, L. Ray, and U. van Kolck, Phys. Rev. C53, 2086 (1996), hep-ph/9511380

[7] N. Kaiser, Phys. Rev. C61, 014003 (2000), nucl-th/9910044 C62, 024001 (2000), nucl-th/9912054

[8] E. Epelbaum et al., Phys. Rev. C65, 044001 (2002), nucl-th/0106007

[9] E. Epelbaum et al., Phys. Rev. C66, 064001 (2002), nucl-th/0208023

[10] A. Nogga et al., Nucl. Phys. A737, 236 (2004).

[11] P. Navratil et al., (2007), nucl-th/0701038.

[12] J. Ley et al., Phys. Rev. C73, 064001 (2006).

[13] H. Witala et al., Phys. Rev. C73, 044004 (2006), nucl-th/0601075

[14] S. Kistryn et al., Phys. Rev. C72, 044006 (2005), nucl-ex/0508012

[15] C. Duweke et al., Phys. Rev. C71, 054003 (2005), nucl-ex/0412024

[16] A. Nogga et al., Phys. Rev. C73, 064002 (2006), nucl-th/0511082

[17] R. Machleidt, Phys. Rev. C63, 024001 (2001), nucl-th/0006014

[18] S. A. Coon and H. K. Han, Few Body Syst. 30, 131 (2001), nucl-th/0101003

[19] A. Deltuva, A. C. Fonseca, and P. U. Sauer, Phys. Rev. C72, 054004 (2005), nucl-th/0509034

[20] E. Epelbaum et al., Phys. Rev. Lett. 86, 4787 (2001), nucl-th/0007057

[21] E. Epelbaum, Prog. Part. Nucl. Phys. 57, 654 (2006), nucl-th/0509032

[22] P. F. Bedaque and U. van Kolck, Ann. Rev. Nucl. Part. Sci. 52, 339 (2002), nucl-th/0203055

[23] S. Ishikawa and M. R. Robilotta, Phys. Rev. C76, 014006 (2007), arXiv:0704.0711 [nucl-th].

[24] S. A. Coon and J. L. Friar, Phys. Rev. C34, 1060 (1986).

[25] R. B. Wiringa, V. G. J. Stoks, and R. Schiavilla, Phys. Rev. C51, 38 (1995), nucl-th/9408016

[26] E. Epelbaum, Phys. Lett. B639, 456 (2006).

[27] D. Rozpedzik et al., Acta Phys. Polon. B37, 2889 (2006), nucl-th/0606017.

[28] V. Bernard, N. Kaiser, and U.-G. Meißner, Nucl. Phys. A615, 483 (1997), hep-ph/9611253

[29] N. Kaiser, R. Brockmann, and W. Weise, Nucl. Phys. A625, 758 (1997), nucl-th/9706045

[30] E. Epelbaum and U.-G. Meißner, Phys. Rev. C72, 044001 (2005), nucl-th/0502052

[31] T. R. Hemmert, B. R. Holstein, and J. Kambor, J. Phys. G24, 1831 (1998), hep-ph/9712496

[32] N. Kaiser, S. Gerstendorfer, and W. Weise, Nucl. Phys. A637, 395 (1998), nucl-th/9802071

[33] H. Krebs, E. Epelbaum, and U.-G. Meißner, Eur. Phys. J. A32, 127 (2007), nucl-th/0703087

[34] V. G. J. Stoks et al., Phys. Rev. C48, 792 (1993). 
[35] SAID on-line program, R. A.Arndt et al., http://gwdac.phys.gwu.edu

[36] B. Borasoy et al., Eur. Phys. J. A31, 105 (2007), nucl-th/0611087

[37] B. Borasoy et al., Nucl. Phys. A768, 179 (2006), nucl-th/0510047

[38] B. Borasoy et al., (2007), arXiv:0708.1780 [nucl-th]. 